\title{
Resampling of hypotheses after negative instances
}

\author{
IRWIN D. NAHINSKY, REBECCA L. HOLLYFIELD, and DAVID E. OESCHGER \\ University of Louisville, College of Arts and Science, Louisville, Kentucky 40208
}

\begin{abstract}
Ninety subjects were run under each of three conditions in a conjunctive concept identification experiment. For one problem, a subject was presented a repetition of the initial positive instance on the trial on which resampling would occur if the subject had not started with the correct hypothesis. For another problem, the subject was presented a new positive instance on that trial; and for a third problem, he was presented a negative instance on that trial. Hypothesis samples were assessed using blank trials following resampling. It was found that a repetition of the original positive instance and a negative instance resulted in about equal problem difficulty, while presentation of a new positive instance produced the best performance ${ }$ Blank trial assessments corroborated the finding. It was concluded that memory for the initial focus was important in the resampling process.
\end{abstract}

In concept identification $(\mathrm{CI})$, the processes involved when subjects must resample hypotheses (Hs) upon discovering that their present $\mathrm{Hs}$ are untenable have long been a question for $\mathrm{H}$ theory. Certain theorists (Bower \& Trabasso, 1964; Restle, 1962) postulated $\mathrm{H}$ resampling with replacement from the entire pool. Other theorists (e.g., Erickson, 1968; Levine, 1966; Wickens \& Millward, 1971; Williams, 1971) have maintained that memory for prior stimulus information, in one way or another, guides the process. Nahinsky (1970) proposed a model in which resampling after exhaustion of a $\mathrm{H}$ sample on a positive instance is done from a positive instance as a focus, and the focal pool is reduced by previously tested Hs. However, if the $\mathrm{H}$ sample is exhausted on a negative instance, resampling does not occur until the next positive instance.

The present experiment was done to gain insight into the resampling process. Subjects were presented a reception CI task in which they would be expected to resample $\mathrm{Hs}$ on a specified trial if they hadn't sampled the correct $H$. Then, a negative instance, the initial positive instance, or a new positive instance was presented, after which the subject's $H$ pool was assessed. Differential $\mathrm{H}$ patterns for the types of instances were used to provide information about the nature of the resampling process and about storage and retrieval of relevant problem information. A blank trial procedure was used to assess $\mathrm{H}$ samples. Nahinsky and Slaymaker (1969) and Slaymaker (1972) presented evidence that such procedures provide reasonable assessments of a subject's $H$ sampling behavior.

This investigation was supported in part by the U. S. Army Research Institute for the Behavioral and Social Sciences, Grant DAHC 19-74-G-0007 to the first author. Requests for reprints should be sent to Irwin D. Nahinsky, Department of Psychology, Lniversity of Louisville. Louisville. Kentucky 40208.

\section{METHOD}

\section{Subjects}

The subjects were 90 introductory psychology students, naive to $\mathrm{CI}$, who participated in fulfillment of a course requirement.

\begin{abstract}
Materials
Stimuli were varied along four dimensions with three values per dimension: (a) shape: square, circle, or cross; (b) shading of figures: black, striped, or white; (c) nu mber of figures: one, two, or three; and (d) number of borders around the figures: one, two, or three. The stimuli were presented via random-access slide projector on a white screen using 2 -in. slides.
\end{abstract}

\section{Design and Procedure}

Each subject was presented with a series of three two-value conjunctive $\mathrm{CI}$ problems. Each problem started with a positive instance, with feedback for correctness of response, followed by a series of three blank trials that involved no feedback for response correctness. Each blank trial stimulus overlapped the initial positive instance by exactly one pair of dimension values. These three stimuli represented three of the six possible overlapping pairs, with one of the pairs corresponding to the correct $\mathrm{H}$. This blank trial set was followed by either three or five feeback trials, with the last of these trials a negative instance, a repetition of the initial positive instance, or a positive instance different from the initial positive instance. This trial was in turn followed by a series of three blank trials. If the trial involved a negative instance or a repetition of the initial positive instance, the following blank trial series was a repetition of the earlier such series. However, if the trial involved a new positive instance, the blank trial series had the same overlap relationship to this new positive instance as the earlier set had to the initial positive instance, and one of the blank trials represented the correct $\mathrm{H}$. The feedback trials preceding the preblank trial stimulus were all negative instances. All blank trial stimuli were shown in random permutation.

The remaining stimuli were next presented in random permutation until the end of the problem, and stimuli were repeated after exhaustion of the population if necessary. Subjects were run to a criterion of 16 consecutive correct responses, whereupon they were questioned about the correct $H$. If the response was correct, the problem was terminated, and if not, the subject was required to make an additional five consecutive correct responses, whereupon he was again questioned about his $\mathrm{H}$. This five-trial procedure was repeated until the correct $H$ was given. Very few protocols requiring this procedure occurred. Subjects were run to criterion or until the first error after Trial 68.

Two groups of 45 subjects were run: one group with all problems in which three trials intervened between the two blank trial sets and 
one group in which five trials intervened between the two blank trial sets. Each subject received three problems such that there was one problem corresponding to each of the three types of instance preceding the second blank trial set as described above. The three problems had solutions "white circles," "two crosses," and "three figures, two borders." The three solutions and the three preblank-trial stimulus conditions were balanced in a Greco-Latin square with presentation order for each of the two groups. Thus, 15 subjects received each presenation order within each of the between-subject conditions.

Each subject was seated at a table containing a response pane that had two response buttons with a third smaller button between them. The left and right buttons were for negative and positive classitications, respectively, and were so labeled. The smaller button was labeled "next slide," which the subject was instructed to press when ready for the following slide. Above the two response buttons were two red lights labeled "correct" and "incorrect" for appropriate feedback that followed immediately on feedback trials No signal followed blank trial responses. The slide and feedback light (if on) went off as soon as the next-slide button was pressed.

A screen separated the experimenter and subject during the experiment. The subject first was explicitly instructed about the nature of the task via tape recorder. He was assured that the lack of feedback on blank trials had nothing to do with response correctness. The subject then received a brief practice problem consisting of a series of six slides with an initial positive instance. The experiment was then begun.

\section{RESULTS AND DISCUSSION}

Protocols were scored for trial to last error (TLE) as a measure of problem difficulty. The analysis of variance for the Greco-Latin square design revealed no significant effect for the number of stimuli between blank trials variable. Thus, the two squares were pooled for an analysis of one large Greco-Latin square with order, problem solution, and preblank-trial stimulus as the within-subjects variables. The preblank-trial stimulus variable yielded a significant effect, $F(2,174)=4.78, p<.01$, $\mathrm{MSE}=411.74$, with means of $22.27,28.40$, and 31.44 for the different-positive instance, negative instance, and same-positive instance conditions, respectively. A contrast of the different-positive instance condition with the other two conditions combined proved significant, $F(1,174)=8.53, p<.01$. Presentation of a positive instance, different from the initial positive instance, on the potential resampling trial produced performance superior to that for a negative instance or a repetition of the initial focus. It seems reasonable to infer from this outcome that subjects use memory for the first positive instance when they resample. Thus, if subjects are presented with the new positive instance, they may use the intersection of this instance with the initial positive instance to reduce greatly the pool of potentially correct Hs. However, repetition of the initial positive instance on the resampling trial would require the subject to refocus on the same stimulus that started the problem, and he would, in effect, start anew. If resampling takes place without memory for prior stimulus information, the two positive instance conditions should have produced the same result. Comparison of the negative instance condition with the same-positive instance condition did not produce a significant difference. Thus, when subjects were presented with an informationally sparse negative instance on resampling, their efficiency is the same as that exhibited on repetition of the initial focus, corroborating the notion that subjects store potential solution information to be used on resampling.

Examination of blank trial patterns after the resampling trial provided further evidence for the proposed resampling process. It was assumed that a positive response to a stimulus that contained a value pair overlapping the preceding feedback positive instance (or the initial positive instance in the case of a preceding negative instance) indicated that the subject had sampled that value pair as a $H$. The first blank trial set was examined to ascertain if the subject had sampled the correct H. Data for the second blank trial set were then tabulated for all subject problems for which the correct $\mathrm{H}$ had not been sampled on the initial positive instance. These protocols represented cases in which resampling should have been required under assumptions of a focusing strategy. The relative frequency with with the correct $\mathrm{H}$ was sampled on the second blank trial set was tabulated for each of the preblank-trial stimulus conditions. The three conditions differed significantly in relative frequency, $\chi^{2}(2)=8.29, p<.025$, with proportions of $.40, .50$, and .75 for the negative instance, same-positive instance, and different-positive instance, respectively. A comparison of the first two conditions with each other produced no significant difference, while comparison of these two conditions combined with the different-positive instance condition yielded a significant difference, $\chi^{2}(1)=7.74, p<.025$. Hence, the likelihood of sampling the correct $H$ in a resampling situation is enhanced by availability of a positive instance along with some prior positive instance. The trend for these results is in line with that for the TLE data.

Finally, an analysis of variance was performed for the preceding second-blank-trial set data, using preblank-trial stimulus as the independent variable and number of positive responses, an indicant of $\mathrm{H}$ sample size, as the dependent variable. The effect was marginally significant, $F(2,161)=2.405, p<.10$, with means of $.473, .678$, and .780 for the negative instance, same-positive instance, and differentpositive instance conditions, respectively. (It should be noted that this analysis, as well as preceding blank-trial analyses, tends to provide a conservative test in view of the partial matching of conditions over subjects that could not be accounted for.) Newman-Keuls tests showed significance only for the negative instance-different positive instance comparison, $q=3.08, p<.05$. (An attempt to contrast the different-positive instance condition with the other two conditions combined resulted in nonsignificance.) The trend of means indicates a general tendency for sample size to be larger on resampling on a positive 
instance than on a negative instance. This outcome may reflect imperfect memory of the initial focus. When a positive instance was presented for resampling, subjects could resample from this new focus. Since this stimulus and the initial focus both contained the correct $\mathrm{H}$, its sampling probability should have been enhanced. On the other hand, negative instance presentation would have required memory for the initial focus.

The results of the experiment suggest that memory for key stimuli plays an important part in $\mathrm{H}$ sampling and that subjects store earlier stimulus information to combine with current information when resampling of $\mathrm{Hs}$ is required. Modification of current $\mathrm{H}$ sampling models may be required. In particular, a modification of Nahinsky's (1970) resampling axioms is called for to account for use of the focus when resampling on a negative instance is required.

\section{REFERENCES}

Bower, G., \& Trabasso, T. Concept identification. In
R. C. Atkinson (Ed.), Studies in mathematical psychology. Stanford, Stanford University Press, 1964.

Erickson, J. R. Hypothesis sampling in concept identification. Journal of Experimental Psychology, 1968, 76, 12-18.

LEVINE, M. Hypothesis behavior by humans during discrimination learning. Journal of Experimental Psychology, 1966, 71, 331-338.

NAHINSKY, I. D. A hypothesis sampling model for conjunctive concept identification. Journal of Mathematical Psychology, 1970, 7. 293-316.

Nahinsky, I. D., \& Slaymaker, F. L. Sampling without replacement and information processing following correct responses in concept identification. Journal of Experimental Psychology, 1969, 80, 475-482.

Restle, F. The selection of strategies in cue learning. Psychological Review, 1962, 69, 329-343.

Slaymaker, F. L. Blank trial effects in concept identification. Journal of Experimental Psychology, 1972, 92, 49-52.

Wickens, T. D., \& Millward, R. B. Attribute elimination strategies for concept identification with practiced subjects. Journal of Mathematical Psychology, 1971, 8, 453-480.

Williams, G. F. A model of memory in concept learning. Cognitive Psychology. 1971, 2. 158-184.

(Received for publication August 8, 1975.) 